\title{
A Tunable Ferrofluid-based Polydimethylsiloxane (PDMS) Microchannel Inductor for Ultra High Frequency Applications
}

\author{
Ahmad Hafiz Mohamad Razy ${ }^{1}$, Mohd Tafir Mustaffa ${ }^{2}$, Asrulnizam Abd Manaf ${ }^{3}$, Norlaili Mohd Noh ${ }^{4}$ \\ ${ }_{1,2,3,4}$ School of Electrical and Electronic Engineering, Universiti Sains Malaysia, Engineering Campus, 14300, \\ Nibong Tebal, Pulau Pinang, Malaysia \\ ${ }^{3}$ Collaborative Microelectronic Design Excellence Centre (CEDEC), Universiti Sains Malaysia, 11900, \\ Bayan Lepas, Pulau Pinang, Malaysia
}

\section{Article Info \\ Article history: \\ Received Jan 3, 2017 \\ Revised Mar 5, 2017 \\ Accepted Mar 20, 2017 \\ Keyword: \\ Ferrofluid-based \\ High tuning range \\ Polydimethysiloxane \\ Quality factor \\ Solenoid inductor \\ Ultra high frequency}

\begin{abstract}
In this work, a tunable ferrofluid-based polydimethylsiloxane (PDMS) microchannel inductor with high quality factor and high tuning range is proposed. For this project, PDMS is used to create a microchannel with a width and height of $0.53 \mathrm{~mm}$ and $0.2 \mathrm{~mm}$ respectively. The microchannel is then used to cover the whole design of a solenoid inductor. A solenoid inductor is designed using wire bonding technique where lines of copper and bond wires are used to form a solenoid winding on top of silicon substrate. A light hydrocarbon based ferrofluid EMG $901660 \mathrm{mT}$ with high permeability of 5.4 is used. The ferrofluid-based liquid is injected into the channel to enhance the performance of a quality factor. A 3D full-wave electromagnetic fields tool, ANSYS HFSS is used in this work to simulate the solenoid inductor. The results obtained in this work gives a quality factor of more than 10 at a frequency range of $300 \mathrm{MHz}$ to $3.3 \mathrm{GHz}$ (Ultra High Frequency range). The highest quality factor is 37 which occurs at a frequency of $1.5 \mathrm{GHz}$, provides a high tuning range of $112 \%$.
\end{abstract}

Copyright @ 2017 Institute of Advanced Engineering and Science. All rights reserved.

\section{Corresponding Author:}

Ahmad Hafiz Mohamad Razy,

School of Electrical and Electronic Engineering,

Universiti Sains Malaysia, Engineering Campus,

14300, Nibong Tebal, Pulau Pinang, Malaysia.

Email: lel.hafiz@gmail.com

\section{INTRODUCTION}

Recently, researches on a tunable MEMS inductors are widely found in literatures. A tunable MEMS inductor provides a broad range of functions especially in RF and microwaves device applications such as radio frequency (RF) power amplifiers, low noise amplifier (LNA) and voltage-controlled oscillator (VCO) [1]. Commonly, tuning configuration of the inductors can be divided into discretes and continuous variable inductors. Both approaches have different functionality, for example, a discrete tunable inductor is often developed using microrelays or microswitches which are used for controlling the length of the inductor coils [2], [3]. For continuous tunable inductor, a method of displacing the magnetic cores of the solenoid inductor is done in order to have a variation of inductance values [3]. Both methods are seen to have a better robustness and design flexibility compare to a tunable capacitor. For that reason, current researches are focusing on tunable inductor for Ultra High Frequency (UHF) applications.

However, the design of tunable inductor(s) is hard to be realized especially at giga-hertz $(\mathrm{GHz})$ range [4]. The difficulty is caused by a relatively smaller size of inductor is required at such frequency range. Plus, with the requirement of high quality factor and good tuning range makes it harder for the design to be realized especially on silicon substrate. On top of that, minituarization of inductor is more difficult due to an ohmic losses and eddy-current losses in a metal traces. Other than that, skin effect also plays its part at high frequency. Most of the current are having a tendency to flow at the outer surface of the conductor. This effect 
will eventually decrease the current density at the centre of a metal conductor and increase the current density near the edge. Hence, a poor quality factor is achieved due to the increase of series resistance of conductor metal [5].

Thus, in this work, new methods are studied to observe the effectiveness of design approaches for gaining a high quality factor for wireless device applications. To achieve a high quality factor alongside tuning capability, a continuous tunable MEMS inductor is proposed. PDMS is used in this project to achieve a configurable tuning for inductance values. The PDMS in this project is used to create a channel for a ferrofluid-based liquid to flow into it and across the solenoid inductor. A high permeability of ferrofluidbased liquid will significantly improve the quality factor of the inductor design [6]. Instead of using a normal approach, a new technique of designing the solenoid inductor is proposed. A wire bonding technique is used in this work to integrate with PDMS. This technique is presented in such a way that lines of copper and bond wires, connected to form a winding solenoid inductor on top of silicon substrate.

\section{RESEARCH METHOD}

In this project, two parts of the inductor designs will be explained. The first part is the PDMS fabrication process. This is important for the design as it will be used to provide the channel for ferrofluidbased liquid to flow across the solenoid MEMS inductor. Specifications of PDMS are explained for a clear view in dimensions perspective. The second part is the design of the solenoid MEMS inductor. After that, the solenoid MEMS inductor will be stacked together with PDMS to obtain a continuous tunable MEMS inductor. A pros and cons of using wire bonding technique also explained in this section. The simulation was carried out by using a 3D full-wave electromagnetic fields tool, ANSYS HFSS where the results of inductance values, quality factor and tuning range were recorded and analysed.

PDMS is chosen in this work because of its characteristic of having a low relative permittivity of 2.3. This advantage will not influence the magnetic field of the solenoid inductor which is crucial for obtaining a high quality factor. The PDMS channel is designed with a length, width and height of $14.8 \mathrm{~mm}$, $0.53 \mathrm{~mm}$ and $0.2 \mathrm{~mm}$ respectively (Figure 1). The overall length, width and height of PDMS are $18 \mathrm{~mm}$, $3 \mathrm{~mm}$ and $2 \mathrm{~mm}$ respectively. Both end of the PDMS has a diameter of $1 \mathrm{~mm}$ which are used for injection of the ferrofluid-based liquid into the channel. A gap of $1 \mathrm{~mm}$ from the holes to the edge of PDMS at each side is important in order to avoid any cracks and leakages from the ferrofluid liquid. In Figure 2(a), the PDMS was stacked at the top of silicon substrate. In Figure 2(b), the solenoid inductor is placed inside of the channel. This method is better compare to microtubing because it will increase the electromagnetic field around the solenoid inductor and hence increase the quality factor of the device. Unlike microtubing, a wall of tubes will eventually reduce the effect of electromagnetic field due to a gap distance between bond wires and magnetic liquid inside the tube.

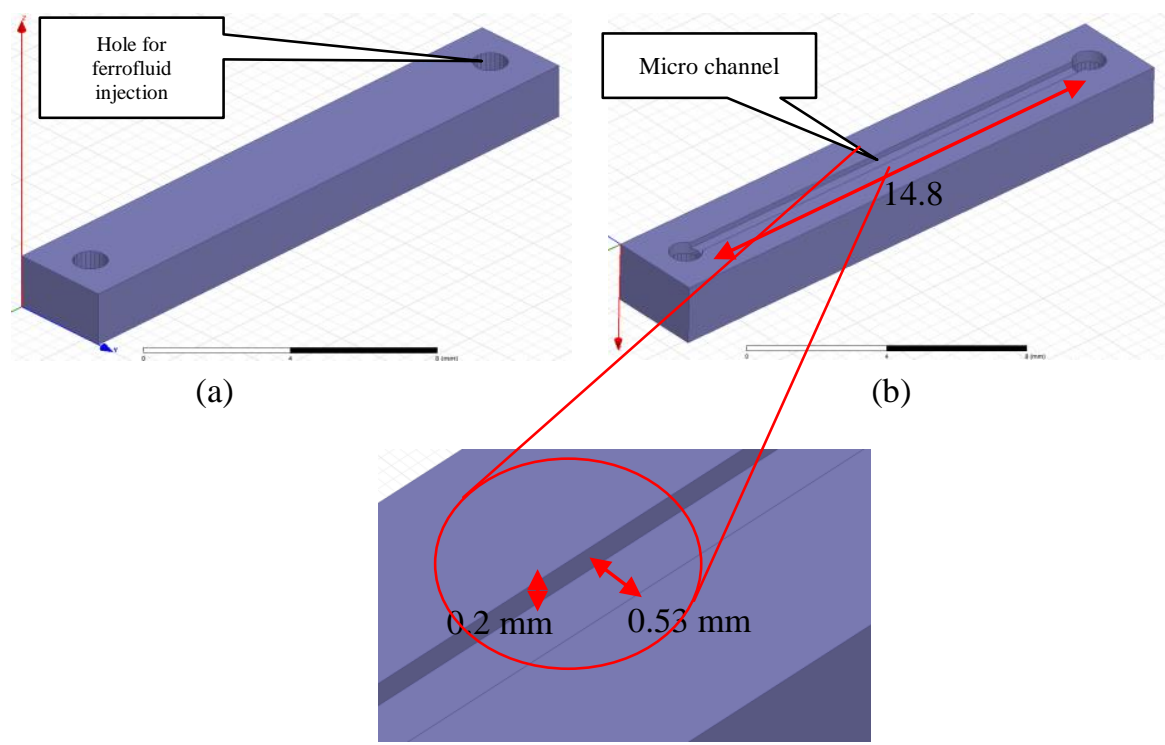

(c)

Figure 1. Top view (a), bottom view (b) and close view (c) of PDMS 


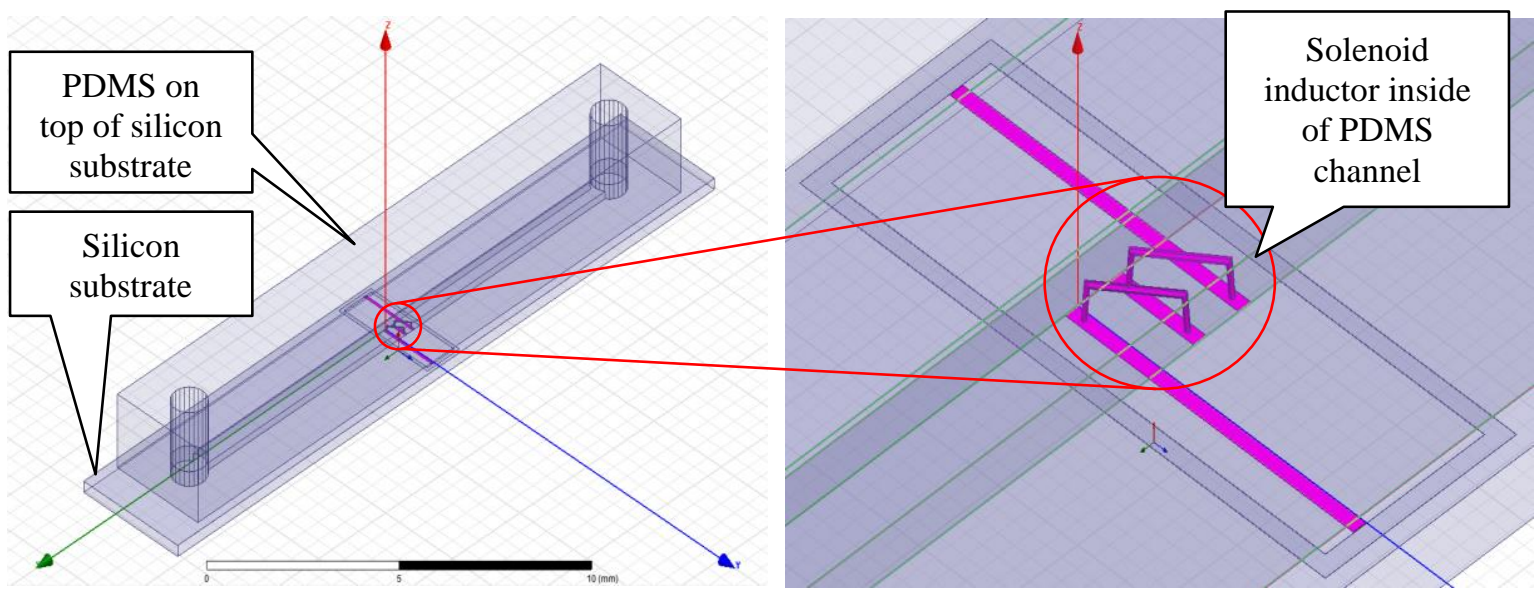

(a)

(b)

Figure 2. Full view of PDMS on top of silicon substrate (a) and close view of solenoid MEMS inductor inside of PDMS channel (b)

In a second part, a technique known as wire bonding is presented here. A basic principle of inductor state that when a normal coil of wire is wrapped around a magnetic material, a magnetic field is produced when the current flows through it. By using the same principle, numbers of copper line patterned on a silicon substrate are bonded together with bond wires to form a winding solenoid inductor. The bond wires will act as a bridge for a current to flows from the first copper line to the second copper line. This method is compatible with the PDMS because the channel of PDMS can fit the whole design of the solenoid inductor (Figure 2 (b)). The length, width and height of bond wires were calculated by using equation (1). According to equation (1), the inductance value can be estimated by modifying the number of turns, sizes of the solenoid inductor and the permeability of the magnetic liquid i.e. ferrofluid-based.

$$
L=\left(\frac{\mu_{0} \times \mu_{r} \times N^{2} \times w \times t}{l}\right)
$$

where $\mu_{0}$ and $\mu_{r}$ representing vacuum permeability $\left(4 \pi \times 10^{-7} \mathrm{H}\right)$ and ferrofluid permeability $(5.4 \mathrm{H} / \mathrm{m}) . N$ is the number of turns of winding coils. $w, t$ and $l$ are the width, thickness and length of copper coils.

In this case, width, thickness and length are representing the copper lines and bond wires. To obtain a $\mathrm{GHz}$ frequency of solenoid inductor, a number of losses need to be examined. The losses such as series resistance, ohmic losses, eddy current effect and skin effect are tend to increase when the number of turns are increases. For this project, number of turns of three is used. This is to ensure the number of losses can be reduced. To achieve a high quality factor, equation (2) is used.

$$
Q=\left(\frac{\omega L}{R_{S}}\right)
$$

where $\omega, L$ and $R_{s}$ are the radian frequency, inductance value and series resistance of copper line. From equation (2), series resistance need to be at a minimal so that a high quality factor can be achieved.

$$
\begin{aligned}
& L=\left(-\frac{1}{2 \times \pi \times f \times i m(Y 11)}\right) \\
& Q=\frac{\operatorname{Im}(Y 11)}{\operatorname{Re}(Y 11)}
\end{aligned}
$$

For that, the inductance values and quality factors were calculated by using formula (3) and (4).

Eventhough the use of the proposed technique gives an extra advantage on its capability to use alongside PDMS, a very high frequency i.e. $5 \mathrm{GHz}$ and above are still hard to be achieved because the size of copper lines need to be much smaller than a current copper width of $80 \mu \mathrm{m}$. Another factor of the downside of using this technique is the silicon substrate issue. A dielectric constant of the silicon substrate is 11.9 
which are higher than both SR4 and Duriod. This will eventually increase the effect of substrate losses of the solenoid inductor and hence reduce the quality factor of the device.

\section{RESULTS AND ANALYSIS}

In this work, simulation were done using a simulation tools ANSYS HFSS. The results of the quality factor and tuning range are presented here. A frequency range of $300 \mathrm{MHz}$ to $3.3 \mathrm{GHz}$ (UHF range) are selected to observe the difference on quality factor performance. A discussion on the results is given to support the theory stated in the previous section. The design is conducted in such a way that the ferrofluidbased liquid is injected into the hole of PDMS. The observations are taken by using a number of step of $0 \%$ (empty channel), $50 \%$ and $100 \%$ (fully-injected channel). A variation of quality factor is observed from the difference levels of ferrofluid-based injection. The tuning range was then calculated based on the results of inductance values obtained.

\subsection{Simulation Results}

In Figure 3, the EM simulation results show that during a step of $0 \%$ (empty channel) to $100 \%$ (fully-injected channel), the quality factor increases from 7 (empty channel) to 12 (fully-injected channel) at frequency of $300 \mathrm{MHz}$ while decreases from 20 (empty channel) to 10 (fully-injected channel) at frequency of $3.3 \mathrm{GHz}$. The results also show that, the opposite changes in a variation of quality factor are due to the self-resonance frequency (SRF). The SRF is shifted from frequency of $5.78 \mathrm{GHz}$ (empty channel) to frequency of $3.96 \mathrm{GHz}$ (fully-injected channel). The SRF is shifted towards a lower frequency due to a heavy number of losses that took place on the device when the magnetic liquid was applied. This losses are caused by the parasitic capacitance, distributed capacitance, floating capacitance and stray capacitance. During a fully-injected channel, the quality factor reached its maximum value of 37 when frequency is at $1.5 \mathrm{GHz}$. After that, the quality factor starts to degrade while moving towards the SRF from its highest value. Therefore, the solenoid inductor designed for this work is having an optimum performance at frequency of $1.5 \mathrm{GHz}$.

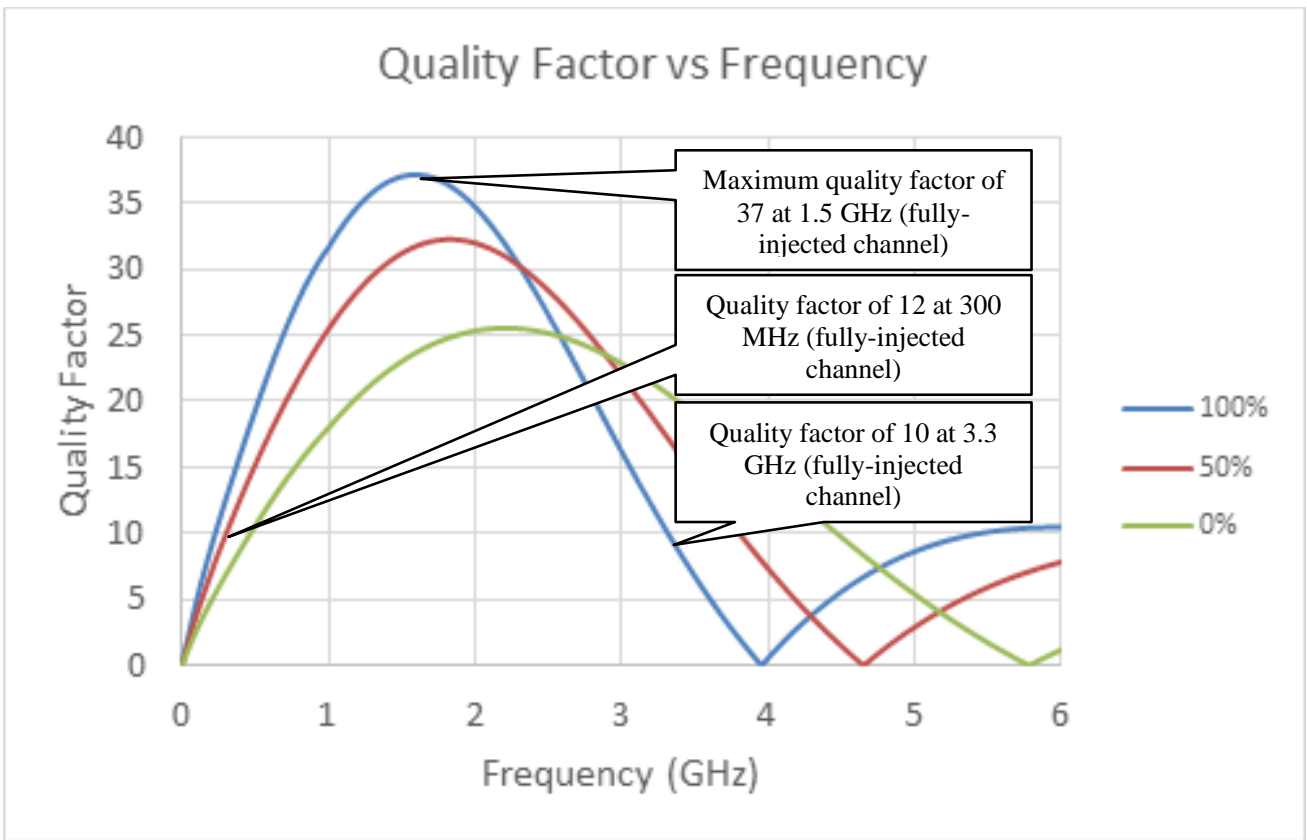

Figure 3. Quality Factor with a difference level of injected ferrofluid

Meanwhile, in Figure 4, the simulation results show that the inductance values changed from minimum to maximum values of $3.86 \mathrm{nH}$ (empty channel) to $8.19 \mathrm{nH}$ (fully-injected channel) at frequency of 1.5 GHz. From observation, an inductance value shows a trend to increase when the inductor operates near the SRF. Therefore, the difference in the inductance values are due to the changes of SRF from empty channel to fully-injected channel. In Figure 5, the graph demonstrates the tuning range of the inductance values from a frequency range of $300 \mathrm{MHz}$ to $3 \mathrm{GHz}$. From the graph, a high tuning range of $112 \%$ at optimum frequency of $1.5 \mathrm{GHz}$ is observed. 


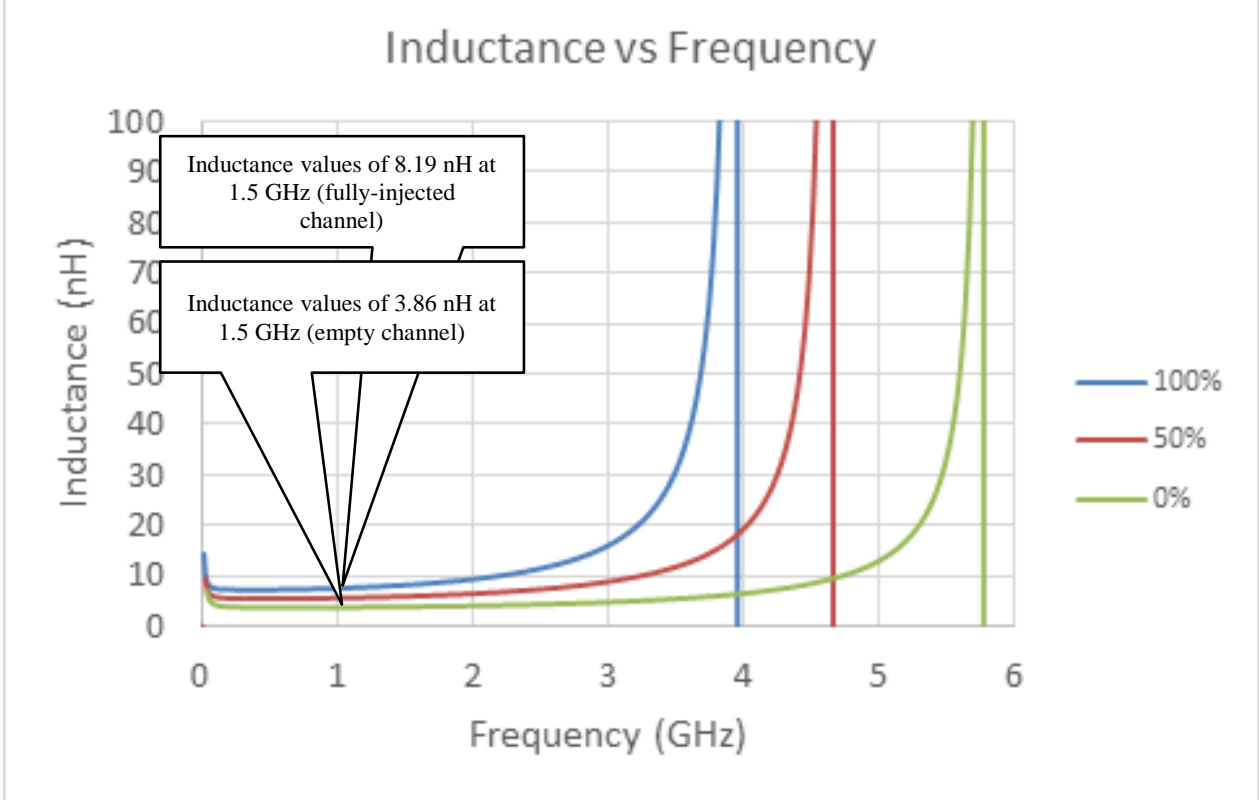

Figure 4. Inductance values with a difference level of injected ferrofluid

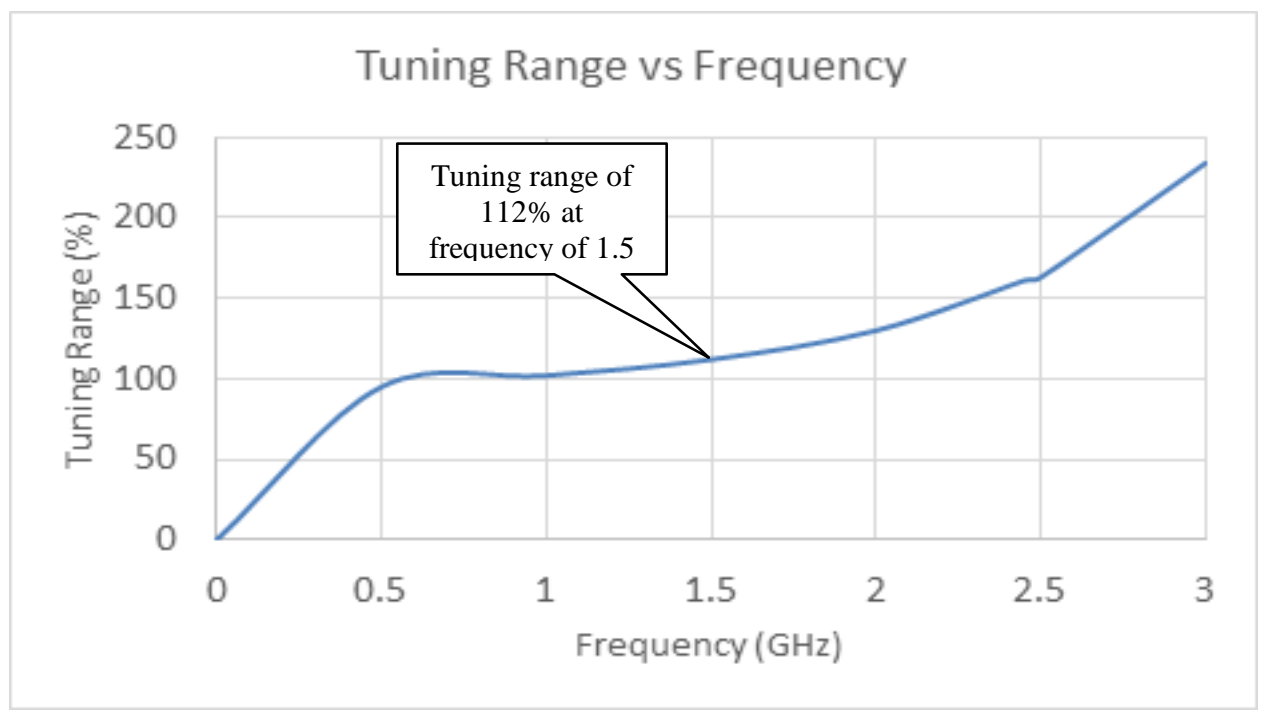

Figure 5. Tuning Range

\subsection{Comparison with State of The Art}

Table 1 shows comparison of this works versus several previous works. The quality factor for this work is considered to be the highest compared to the other works. For tuning range, the designed inductor is better than [7] but some of previous works are not tunable.

Table 1. Comparison in terms of performance with previous works

\begin{tabular}{ccccc}
\hline Reference No. & Frequency $(\mathrm{GHz})$ & Inductance Values $(\mathrm{nH})$ & Quality Factor & Tuning Range $(\%)$ \\
\hline This work & 1.5 & $3.86-8.19$ & 37 & 112 \\
{$[7]$} & $1.0-5.0$ & $5.5-6.8$ & 34 & 24 \\
{$[8]$} & 2.4 & 1.95 & 9.7 & - \\
{$[9]$} & 5.23 & 0.61 & 27.31 & - \\
{$[10]$} & 2.5 & 4.6 & 3.1 & - \\
{$[11]$} & 2.4 & 2.67 & 16.7 & - \\
\hline
\end{tabular}




\title{
4. CONCLUSION
}

The aims of this work was to design the solenoid MEMS inductor using the PDMS material for UHF applications. The inductor was designed to achieve a high quality factor and a high tuning range at a frequency range of $300 \mathrm{MHz}$ to $3 \mathrm{GHz}$. This project utilized PDMS material which allows any desired width and height of the channel. A light hydrocarbon based ferrofluid EMG $901660 \mathrm{mT}$ was used in such a way that the ferrofluid-based liquid can be injected into the PDMS channel. The ferrofluid-based liquid has a high permeability of 5.4 which can increase the performance of the quality factor of the solenoid MEMS inductor. This work was conducted by injecting the ferrofluid-based liquid into the PDMS channel (through simulation) by a step of $0 \%$ (empty channel), $50 \%$ and $100 \%$ (fully-injected channel). From simulation, the quality factor of more than 10 was obtained at the desired UHF range. The maximum quality factor of 37 was achieved at frequency of $1.5 \mathrm{GHz}$. Besides, the results demonstrate a successful high tuning range of $112 \%$ at the optimum frequency of $1.5 \mathrm{GHz}$.

\section{ACKNOWLEDGEMENTS}

This work is sponsored by MOHE Exploratory Research Grant Scheme (ERGS), grant number (203/PELECT/6730112).

\section{REFERENCES}

[1] N. Ning, et al., "A tunable magnetic inductor," IEEE Trans Magn., 2006.

[2] D. M. Fang, et al., "A review of the tunable microinductors".

[3] B. Assadsangabi, et al., "Ferrofluid-based variable inductor," Proc IEEE Int Conf Micro Electro Mech Syst., vol. 2, pp. 1121-4, 2012.

[4] B. Azzerboni, et al., "Magnetic Nanostructures in Modern Technology," Netherlands, Springer, 2008.

[5] C. Bowick, et al., "RF circuit design," $2^{\text {nd }}$ Edition, USA, Newnes, 2008.

[6] F. Banitorfian, et al., "A Novel Tunable Water-Based RF MEMS Solenoid Inductor," Micro Nanoelectron (RSM), IEEE Reg Symp., pp. 58-61, 2013.

[7] N. Sarkar, et al., "Microassembled tunable MEMS inductor," 18th IEEE Int Conf Micro Electro Mech Syst., 2005. MEMS 2005, pp. 183-6, 2005.

[8] S. Seok, et al., "A High Performance Solenoid-type MEMS Inductor," vol/issue: 1(3), pp. 1-7, 2001

[9] P. Pirouznia, B. A. Ganji, "Analytical Optimization of High Performance and High Quality Factor MEMS Spiral Inductor," vol. 34, pp. 171-9, 2014.

[10] R. L. Bunch, et al., "Quality factor and inductance in differential IC implementations," IEEE Microw Mag., vol/issue: 3(2), pp. 2-7, 2002.

[11] H. J. D. L. Santos, "On the ultimate limits of IC inductors-an RF MEMS perspective," 52nd Electron Components Technol Conf., (Cat No02CH37345), vol/issue: 3(1), pp. 1027-31, 2002.

\section{BIOGRAPHIES OF AUTHORS}

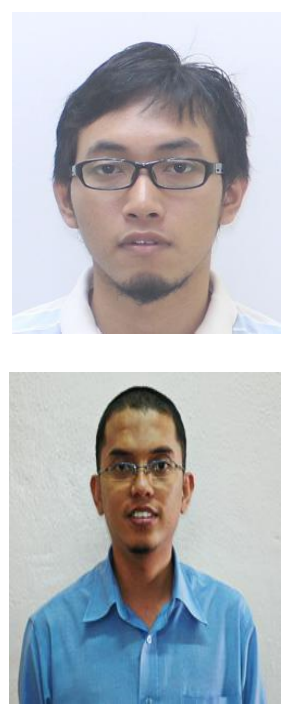

Ahmad Hafiz Mohamad Razy graduated in BEng (Electronic) degree from Universiti Sains Malaysia (USM) in 2013. Currently, he is pursuing MSc study in microfluidic-based tunable MEMS inductor at USM.

\begin{abstract}
Mohd Tafir Mustaffa received his BEng degree in electrical and electronic engi-neering from Universiti Sains Malaysia (USM), Penang in 2000. He was awarded a master degree (MEngSc) in computer and microelectronic engineering from Victoria University, Australia in 2005 and was officially completed his $\mathrm{PhD}$ degree in electrical engineering specializing in radio frequency integrated circuit (RFIC) in September 2009. He served as a system engineer at Data Acquisition Sys-tem (M) Sdn. Bhd and tutor in USM. He is now a senior lec-turer at the School of Electrical and Electronic Engineering, USM, Engineering Campus. Dr Mohd Tafir Mustaffa is a senior member of IEEE, and also a member of Advanced Inte-grated System Device, a group research at USM. He is actively involved with IEEE Circuits and Systems Society for the last few years as a committee member. He is the author and co-author of more than 40 technical papers in conferences and journals, book and book chapters. He is currently involved in the research of digital and analog IC, RFIC, and RF MEMS design.
\end{abstract}






Asrulnizam Abd Manaf received B Eng and MSc degrees in electrical and elec-tronic engineering from Toyohashi Uni-versity of Technology, Japan in 2001 and 2005, respectively. He worked as an elec-trical engineer at the Toyo-Memory Technology Sdn. after completing his undergraduate study. He pursued his PhD study in Keio University, Japan in 2006. He received $\mathrm{PhD}$ in engineering from the Department of Applied Physic and Physico Informatics, School of Fundamen-tal Science and Technology, in 2009. He joined the School of Electrical and Electronic Engineering, Universiti Sains Malay-sia (USM) as a senior lecturer. He was promoted to an associ-ate professor in 2015. He has authored and co-authored 60 international technical journals or conference papers. His cur-rent research interest includes development of microfluidic-based DNA sensor integrated with CMOS circuitry, miniatur-ized of fluidic-based inclination sensor, bio inspired based microfluidic acoustic, pressure and flow sensor for underwater system, micro fluidic-based memristor, micro Thermoelectric Generator (mTEG)based energy harvesting, graphene-based transistor, and micro three-dimensional fabrication technique by using grayscale Technology.

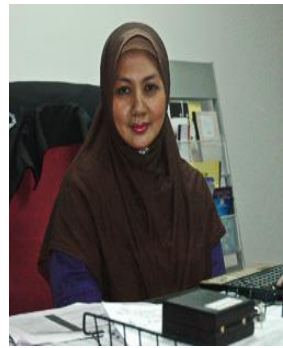

Norlaili Mohd Noh received her BEng degree in electrical engineering from Universiti Teknologi Malaysia (UTM) in 1987, MSc and PhD degrees from Universiti Sains Malaysia (USM) in 1995 and 2009, respectively. She is currently a senior lecturer with the School of Electri-cal and Electronic Engineering USM. She was promoted to an associate professor in 2015. Her current research interests include low-noise amplifier design, analog circuit design, and radio frequency integrated circuit (RFIC) design. 